\title{
The Impact of the Vertical Variation of Cloud Droplet Size on the Estimation of Cloud Liquid Water Path and Its Potential for Rain Detection
}

\author{
Z. Li, R. Chen, and F-L Chang \\ Earth System Science Interdisciplinary Center, University of Maryland \\ College Park, Maryland
}

\section{Introduction}

Liquid water path (LWP) is an important cloud microphysical property that determines the climatic effects of boundary layer clouds. Satellites provide the only means of acquiring global and long-term LWP estimates. The LWP is estimated from satellite measurements of either microwave radiation emitted by the cloud or visible/near infrared (NIR) solar reflectance from the cloud.

LWP can be retrieved from the microwave signature emitted by cloud droplets. Microwave retrievals of cloud LWP are not applicable over land because of the strong and highly variable microwave emission of the land surface. The emission from ocean surfaces is relatively weak and less variable so cloud LWP can be estimated from satellite observed microwave radiances. However, the accuracy is significantly affected by the sea surface temperature, surface wind speed, and atmospheric precipitable water vapor.

Cloud LWP can also be estimated from solar reflectance measurements made during the daytime. In the visible/NIR method, cloud LWP is derived from two other cloud properties: cloud optical depth and droplet effective radius (DER). The retrieval of cloud optical depth utilizes the solar reflectance measurements at a visible channel and the retrieval of DER utilizes the solar reflectance measurements at a NIR channel. However, the DER retrieved with single NIR channel is weighted toward cloud top because of cloud absorption (Platnick 2000). Assuming that the DER has a linear distribution in the vertical direction, Chang and $\mathrm{Li}$ (2002) presented a method to determine an optimal linear-DER profile by using a combination of all three NIR measurements. The profile that is retrieved by the Chang and $\mathrm{Li}$ method estimates the vertical variation of DER and improves the accuracy of the LWP estimation (Chang and Li 2003), which will be demonstrated in this paper. Potential impact of the DER profile on warm rain detection is also discussed. 


\section{Data and Methodology}

Data collected on January 1, 2003 from the Moderate Resolution Imaging Spectroradiometer (MODIS) and the Advanced Microwave Scanning Radiometer (AMSR-E) on the Aqua satellite are used in this investigation. The investigation is limited to warm cloud over tropical oceans $\left(40^{\circ} \mathrm{N} \sim 40^{\circ} \mathrm{S}\right)$. To minimize the impact of cloud 3-dimensional (3D) effects, the satellite viewing angle is limited to $30^{\circ}$ and the solar zenith angle is limited to $50^{\circ}$. To eliminate ice contamination, only warm (cloud-top temperatures $>273 \mathrm{~K}$ ) and water clouds are selected.

\section{MODIS Retrieval}

Traditionally, cloud LWP is derived using retrievals of cloud optical depth, $\tau$, and droplet effective radius, as given by $r_{\epsilon}^{L W P}=4 \rho_{w} / 3 Q_{\text {ext }} \tau r_{e}$, where $\rho_{w}$ is the density of liquid water, is the extinction efficiency. The LWP calculated by this equation assumes that re is vertically constant $Q_{e}$.

In this study, the MODIS $0.86-\mu \mathrm{m}$ reflectance measurement is used to retrieve $\tau$ for clouds over ocean. However, there are three different MODIS NIR channels at $3.7 \mu \mathrm{m}, 2.1 \mu \mathrm{m}$, and $1.6 \mu \mathrm{m}$. Each of the three NIR channels can be used to retrieve a different re, namely, $r_{e} 3.7, r_{e} 2.1$ and $r_{e} 1.6$. The retrieval of $r_{e} 3.7$ follows the method of Chang et al. (2000).

Due to cloud absorption, the DER retrieved from a single NIR channel is mainly sensitive to the layer near the cloud top, which can cause biases in LWP calculations if $r_{e}$ varies vertically. For a cloud with a decreasing DER profile (DDP) with height, that is, a smaller $r_{e}$ towards cloud top, the calculated LWP would be underestimated. On the contrary, for a cloud with an increasing DER profile (IDP), that is, a larger re towards cloud top, the calculated LWP would be overestimated.

To account for the vertical variation in re, Chang and $\mathrm{Li}(2002,2003)$ present a method using combined information from the multi-NIR channels at 3.7,2.1 and 1.6 $\mu \mathrm{m}$ to retrieve a linear re profile and calculate cloud LWP. Here, the linear re profile is parameterized by $r_{e} 1$ at cloud top and $r_{e} 2$ at cloud base. For retrievals of $r_{e} 1$ and $r_{e} 2$, an optimal solution set is determined by match the MODIS measurements with radiative transfer calculations at all three NIR channels, i.e., 3.7, 2.1 and 1.6 $\mu \mathrm{m}$. Figure 1 shows the frequency distributions of the retrieved re 1 and $r_{e} 2$. The spectrum of $r_{e} 2$ is wider than the spectrum of $r_{e} 1$.

To show the effects of the different re on LWP estimations, different LWPs are calculated using $r_{e} 3.7$, $r_{e} 2.1$ and $r_{e} 1.6$ with assumptions of a vertically constant re (hereafter referred to as LWP3.7, LWP2.1, and LWP1.6) and using the linear re profile (hereafter referred to as LWPrep). The root mean square (RMS) difference between LWPrep and LWP3.7 is $0.031 \mathrm{~mm}$, which is about $25 \%$ of the mean value. 


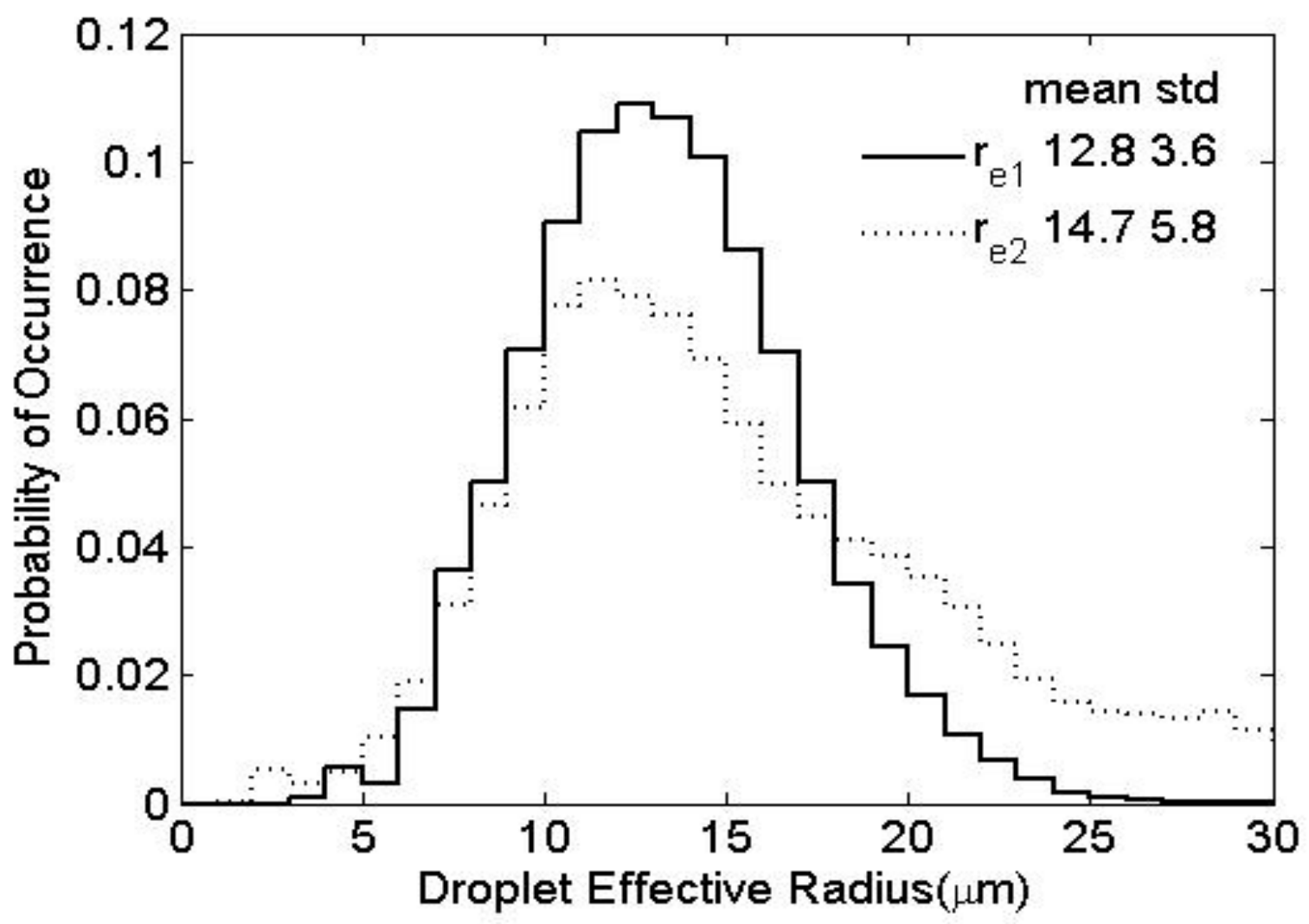

Figure 1. Probability Density Function of the DER retrieved from a combination of three MODIS NIR channels.

LWP1.6 is the closest to LWPrep, but there is still a RMS difference of $0.017 \mathrm{~mm}$. Therefore, vertical variation of cloud DER has a significant impact on the LWP estimations.

Note that four MODIS detectors measuring radiances at $1.6 \mu \mathrm{m}$ are non-functional. The average of measurements made by the nearest live detectors is used here. This should not affect the results much because finally, only overcast clouds are considered in this study.

\section{AMSR-E Retrieval}

The AMSR-E microwave measurements used in this study have 12 channels and 6 frequencies ranging from $6.9 \mathrm{GHz}$ to $89.0 \mathrm{GHz}$. Horizontally and vertically polarized radiation is measured separately at each frequency. The AMSR-E standard ocean algorithm (Ashcroft and Wentz 2000) retrieves sea surface temperature, surface wind, water vapor, and LWP from the signal emitted by surface and atmospheric components at $6.9 \mathrm{GHz}, 10.7 \mathrm{GHz}, 18.7 \mathrm{GHz}$ and $36.5 \mathrm{GHz}$. The algorithm can retrieve LWP when there is no rainfall or if the rain rate is less than $2 \mathrm{~mm} / \mathrm{hr}$. The AMSR-E LWP product is compared with the LWPs derived from the coincident MODIS measurements. The AMSR-E ocean product also provides rain flags, which are used to determine whether a cloud is raining or not. 


\section{Results}

In this study, the MODIS LWP estimates are compared with AMSR-E LWP estimates (LWPMW). The AMSR-E and MODIS are on the same satellite platform of Aqua. The MODIS measurements are matched to the larger AMSR-E footprint according to the navigation data. The statistical relationships between the MODIS and AMSR-E measurements are discussed to show the effect of the DER vertical variation on the MODIS LWP estimation and its potential for rain detection. Overcast clouds with optical depth from 3.6 to 23 are utilized to avoid the microwave background bias and the ice contamination. Figure 2 is a scatter plot of MODIS LWP2.1 as a function of AMSR-E LWPMW.

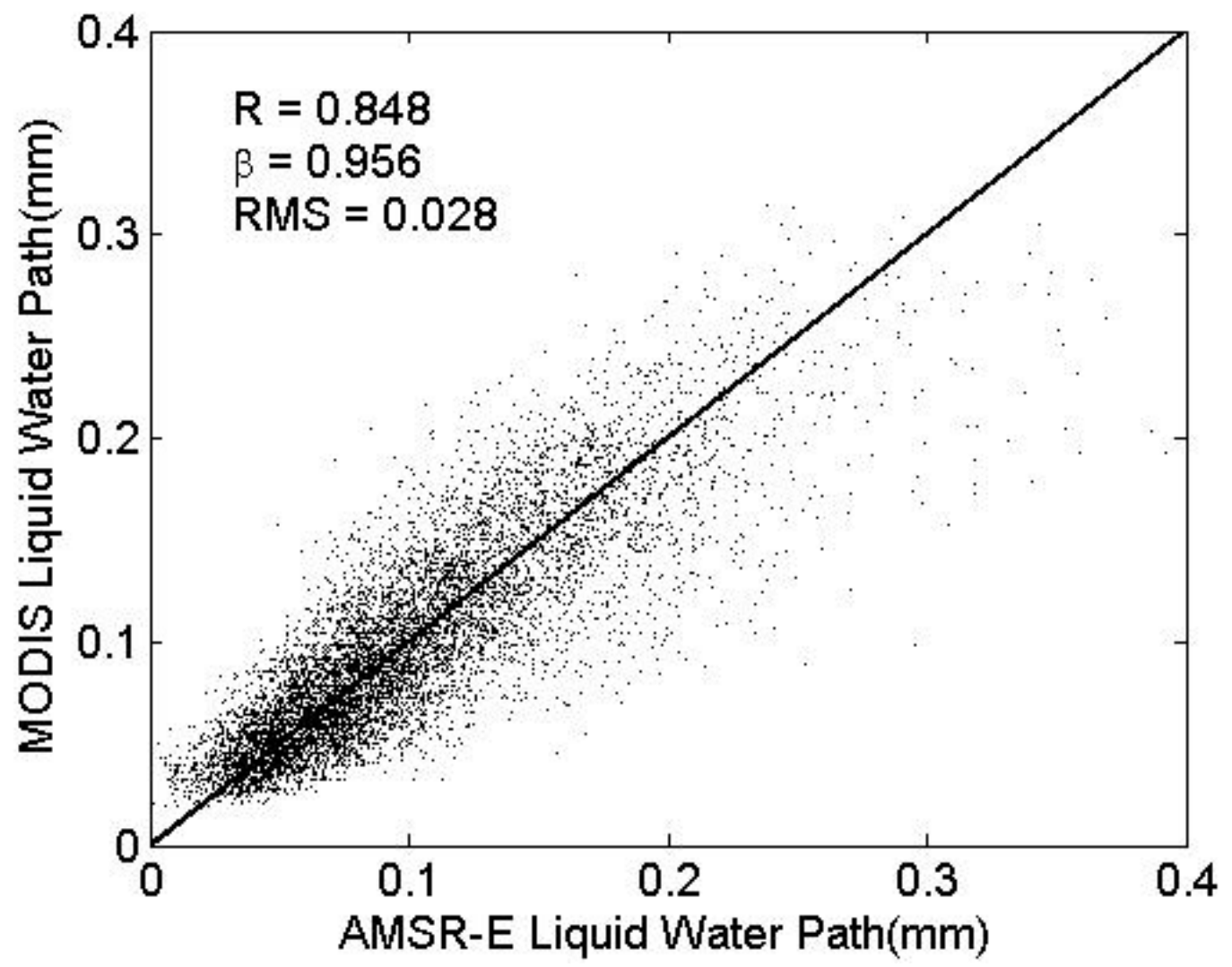

Figure 2. Comparison between AMSR-E LWP and MODIS LWP2.1 R is correlation and $\beta$ is the linear regression coefficient.

\section{Effect of Cloud DER Vertical Variation}

As previously stated, using a vertically constant DER, the LWP is overestimated for clouds with IDP, and is underestimated for clouds with DDP. Because the microwave LWP estimation measures the entire cloud layer, it is utilized to evaluate whether this impact improves LWP estimation or not. Clouds over AMSR-E footprints are separated into three categories: clouds with a neutral DER profile (NDP), IDP clouds and DDP clouds based on $r_{e} 1$ and $r_{e} 2 . r_{e} 2$ is $10 \%$ larger than $r_{e} 1$ for DDP clouds and $10 \%$ 
less than $r_{e} 1$ for IDP clouds. For neutral clouds, the vertical variation of DER is within $10 \%$. Because the vertical variation of DER causes the largest bias in LWP3.7, LWP3.7 is used to illustrate how the DER profile improves LWP estimations. LWP2.1 and LWP1.6 show similar biases of smaller magnitude.

LWP3.7 is $12.6 \%$ larger than LWPMW for IDP clouds, $2.6 \%$ larger than LWPMW for NDP clouds and $11.2 \%$ less than LWPMW for DDP clouds. Since the DER profile is the only criteria separating the data, it must be the primary cause for the differences. LWPrep, LWP3.7, LWP2.1, and LWP1.6 are almost identical for NDP clouds because there are no vertical variations in the DER. The approximate 3\% difference between MODIS LWP estimations and AMSR-E LWP estimations for NDP clouds is due to other uncertainty factors. So over the AMSR-E footprint, the bias caused by the vertical variation of DER in visible/NIR LWP estimations is about $+10 \%$ for IDP clouds and $-14 \%$ for DDP clouds.

LWPrep is 5.2\% larger than LWPMW for IDP clouds and $0.1 \%$ larger than LWPMW for DDP clouds. Both differences are close to the 3\% difference for NDP clouds. This means that the DER profile improves the LWP estimations and corrects the bias caused by the vertical variation of the DER.

\section{Implication for Warm Rain Clouds}

The fundamental theory of cloud physics states that cloud droplet size increases with height during the developing stage due to condensation growth. Once a collision process starts, larger droplets tend to fall to the lower levels of the cloud. Therefore, the DER at cloud base is small for developing clouds and large for drizzling clouds. So the DER at the cloud base is more correlated with rainfall than DER at the cloud top.

Figure 3 shows the distribution of DER at the cloud base and the cloud top for raining and non-raining clouds, respectively, which are defined by the AMSR-E rain flag. Figure 3a shows that raining causes a DER increase of $3.5 \mu \mathrm{m}$ at the cloud top and an increase of $7 \mu \mathrm{m}$ at the cloud base (Figure 3b). So DER at the cloud base is more effective for rain detection. For example, if we define a threshold of $14 \mu \mathrm{m}$ for raining clouds (Rosenfeld and Gutman 1994), the DER at the cloud base can catch $87.0 \%$ of AMSR-E detected rains, while the DER at the cloud top only catches $64.4 \%$ of AMSR-E detected rains. For some AMSR-E detected raining clouds, the DER at the cloud base can be as small as $10 \mu \mathrm{m}$. These clouds could be partially raining, while overall small DER is evident because of the effect of the non-raining part of the clouds. However, based on the same $14 \mu \mathrm{m}$ raining threshold, the false raining detection rate is $22.7 \%$ using the DER at the cloud top and 30.6\% using the cloud bottom DER. If the DER threshold is increased to $20 \mu \mathrm{m}$, the false detection rate is considerably reduced, at the expense of missing some raining clouds. The false detection may be due to the AMSR-E sensitivity problem. Many of these AMSR-E defined non-raining clouds could have very light rain or drizzle which evaporates before reaching the ground. 
a) DER at cloud top

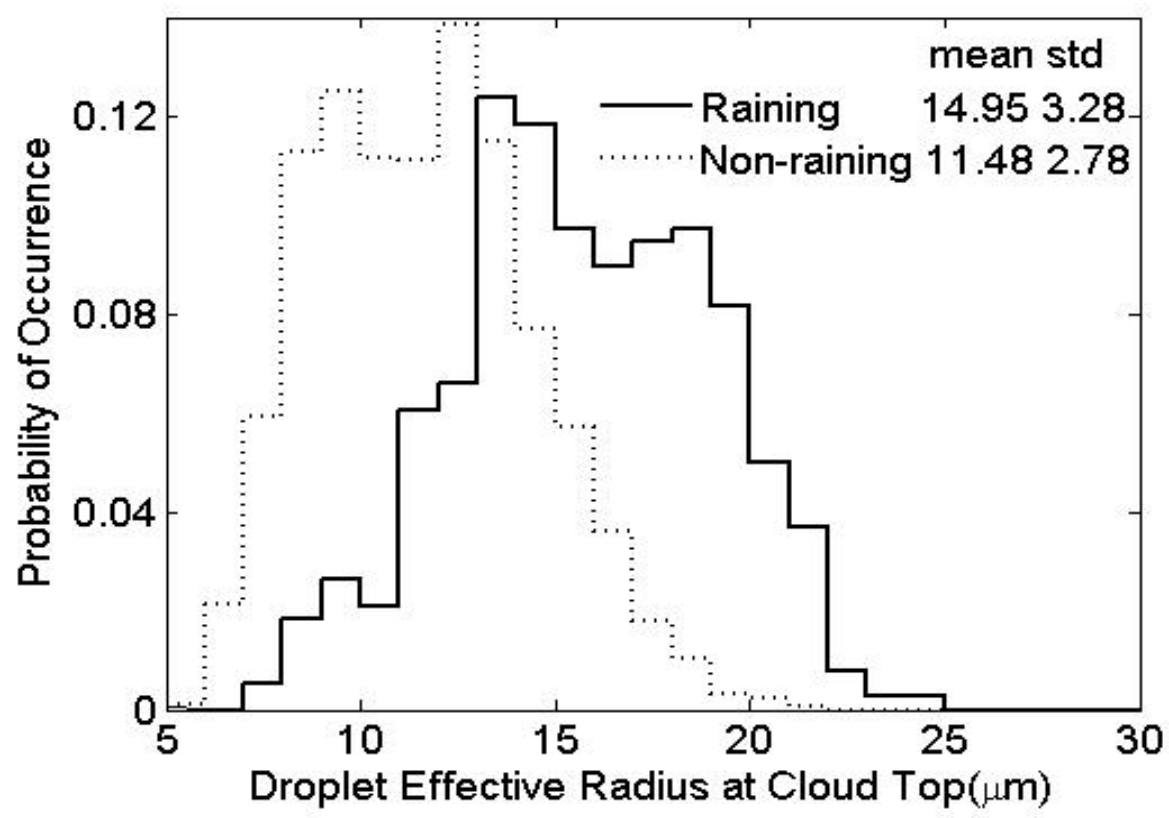

b) DER at cloud base

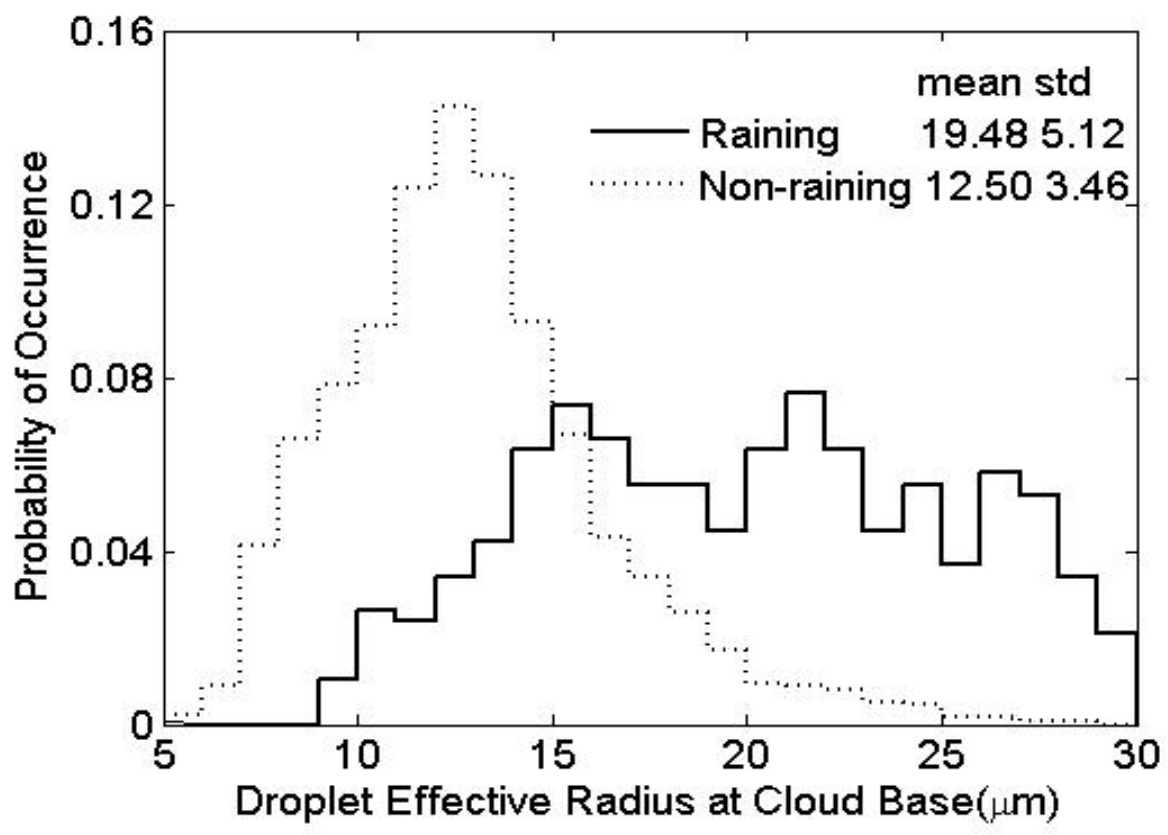

Figure 3. Probability density function of DER at cloud top and cloud base for raining and non-raining cloud. 


\section{Concluding Remarks}

This investigation demonstrates that a substantial bias in the estimation of LWP is incurred by assuming a constant DER. It is shown that DER profiles retrieved using the algorithm of Chang and Li (2002) can remove the bias and improve LWP estimates. These DER profiles can help in detecting warm rain.

\section{Acknowledgment}

This study is supported by the Department of Energy Atmospheric Radiation Measurement program and DEFG0201ER63166 and National Oceanic and Atmospheric Administratin's GOES-R Risk reduction and GOES-R algorithm development programs

\section{Reference}

Ashcroft, P, and FJ Wentz. 2000. AMSR Algorithm Theoretical Basis Document. RSS Technical Report 121599B-1. Page 27.

Chang, F-L, Z Li, and SA Ackerman. 2000. "Examining the relationship between cloud and radiation quantities derived from satellite observations and model calculations." Journal of Climate 13:3842-3859.

Chang, F-L, and Z Li. 2002. "Estimating the vertical variation of cloud droplet effective radius using multispectral near-infrared satellite measurements.” Journal of Geophysical Research 107(AAC 7)1-12.

Chang, F-L, and Z Li. 2003. "Retrieving vertical profiles of water-cloud droplet effective radius: Algorithm modification and preliminary application." Journal of Geophysical Research 108(AAC 3)1-11. 